\title{
The relative effects of crude oil price and exchange rate on petroleum product prices: Evidence from a set of Northern Mediterranean countries ${ }^{\text {is }}$
}

\author{
M. Hakan Berument ${ }^{\mathrm{a}, *}$, Afsin Sahin ${ }^{\mathrm{b}, 1}$, Serkan Sahin ${ }^{\mathrm{c}, 2}$ \\ a Bilkent University, 06800 Ankara, Turkey \\ b Gazi University, 06571 Ankara, Turkey \\ c GlobalData, EC1N 8EB London, United Kingdom
}

\section{A R T I C L E I N F O}

\section{Article history:}

Accepted 3 July 2014

Available online 2 August 2014

\section{Keywords:}

Oil prices

Exchange rates

Asymmetry

\begin{abstract}
A B S T R A C T
This paper provides a set of empirical evidence from five Northern Mediterranean countries that are subject to similar refinery reference prices regarding the relative sensitivity of crude oil prices and exchange rate on (pre-tax) petroleum product prices. The empirical evidence reveals that a one percent increase in exchange rate (depreciation) increases petroleum product prices less than a one percent increase in crude oil prices does in the long run. In the short run, however, a one percent increase in exchange rate increases petroleum product prices more than a one percent increase in crude oil prices does.
\end{abstract}

(C) 2014 Elsevier B.V. All rights reserved.

\section{Introduction}

The two most important determinants of petroleum product prices in local markets are crude oil prices (denominated in US dollars (USD)) and local currency-USD exchange rate. ${ }^{3}$ The purpose of this article is to study the relative pass-through effects of these two costs on petroleum product prices. To be particular, we will assess which of these two input' price changes affect petroleum product prices more. We will focus on how these two costs affect petroleum product prices in the long run as well as in the short run.

Retail petroleum product prices are subject to changes in cost stemming mainly from crude oil prices, exchange rate besides taxes, transportation, and labor. Norman and Shin (1991) and Bachmeier and Griffin (2003) for the US, Kirchgassner and Kubler (1992) for Germany, and Godby et al. (2000) for Canada report the positive effect of (crude) oil prices on gasoline prices. There are a number of studies that report

\footnotetext{
We would like to thank Bülent Hayaloğlu, Rana Nelson, Çağla Ökten and the anonymous referee for their helpful comments.

* Corresponding author. Tel.: +90 312290 2342; fax: +90 3122665140.

E-mail addresses: berument@bilkent.edu.tr (M.H. Berument), afsinsahin@gazi.edu.tr (A. Sahin), ssahin@globaldata.com (S. Sahin).

URL's: http://www.bilkent.edu.tr/ berument (M.H. Berument),

http://websitem.gazi.edu.tr/afsinsahin (A. Sahin).

${ }^{1}$ Tel.: + 903122162116 ; fax: +90312 2162111 .

2 Tel.: + 447535085146 .

3 There are other determinants of petroleum product prices, such as labor cost, transportation cost, and gas station rent. To enable cross-country comparisons, we assume that the contributions of these items to the cost of petroleum product prices are either stable or move with these determinants. Thus, we implictly assume that these determinants move with exchange rates or crude prices.
}

the positive effect of exchange rate on petroleum product prices. Chou (2012), Bacon (1991), Balke et al. (1998), Reilly and Witt (1998), Asplund et al. (2000), and Galeotti et al. (2003) model the role of exchange rate and crude prices for a set of non-US countries. Different types of shocks affect product prices differently. For example, Pindyck (2001) distinguishes between permanent and temporary shocks and Radchenko (2005) decomposes shocks into long term versus short term. Since changing prices is costly, firms are less likely to change product prices if there is more chance that they will have to change their product prices again in the near future, such as if input price increases will correct themselves shortly. Firms tend to increase their product prices more if the shock is permanent (rather than transitory) and long term (rather than short term). These studies suggest that agents will move to adjust to changes differently depending on the type of shocks.

To assess the role of exchange rate on petroleum product prices, we gathered data from a set of countries whose currencies are neither the USD nor fixed to it. We chose a set of Northern Mediterranean (or Southern European) countries that share the same or similar crude oil reference prices and where exchange rate is determined by the markets. Thus, we collected data from France, Greece, Italy, Spain, and Turkey. Section 2 presents the data and Section 3 describes the methodology we employed and discusses the results obtained by the econometric specifications, and Section 4 concludes.

\section{Data}

We employed weekly data from January 03,2005 to October 22, 2012 for France, Greece, Italy and Spain, and from January 01, 2005 to December 25, 2011 for Turkey. For France, Greece, Italy, and Spain we 
gathered consumer prices of petroleum products, with duties and taxes deducted, for Euro-super 95, fuel oil, gas oil for automobiles, and heating gas oil from the Oil Bulletins of the European Commission. For Turkey, we collected the selling prices (vendor) without taxes for diesel, 95 octane unleaded gasoline, and fuel oil from the annual official reports of the Turkish Energy Market Authority. We obtained Brent oil data for world oil prices from Data Stream and gathered the USD-Euro exchange rate from the Federal Reserve Bank of St. Louis and the Turkish Lira-USD exchange rate from the data delivery system of the Central Bank of the Republic of Turkey. All the data employed in this study were used in their logarithmic forms.

\section{Methodology}

To model petroleum product pricing, we follow Campa and Goldberg's (2005) micro foundations of exporters' pricing behavior of import prices. The import price of oil $\left(P_{t}^{m}\right)$ is the product of exchange rate $\left(E_{t}\right)$ and the export price of a product (of an oil export price, $O_{I} L_{t}$ ) $P_{t}^{x}$. The export price (in USD) can be equal to the marginal cost $\left(M C_{t}^{x}\right)$ in USD that captures crude oil prices, exchange rate, and mark-up $\left(M_{A R K U P}^{x}\right)$. If the lower case letters represent the logarithm of the variables, then we can write the import price of oil in Eq. (1) as follows:

$p_{t}^{m}=e_{t}+m c_{t}^{x}+\operatorname{markup}_{t}^{x}$

We further assume that marginal cost is affected by benchmark crude oil prices and that mark-up is affected by exchange rate:

$m c_{t}^{x}=\psi_{0}+\psi_{1}$ oil $_{t}$

$\operatorname{markup}_{t}^{x}=\varphi_{0}+\varphi_{1} e_{t}$

Thus, we may write Eq. (1):

$p_{t}^{m}=\psi_{0}+\varphi_{0}+\psi_{1}$ oil $+\left(1+\varphi_{1}\right) e_{t}$.

In this paper, we are interested in the effect of relative change of exchange rate to crude oil prices on petroleum product prices. Thus, we could write:

$p_{t}^{m}=\psi_{0}+\varphi_{0}+\psi_{1}\left(\right.$ oil $\left._{t}+e_{t}\right)+\left(1+\varphi_{1}-\psi_{1}\right) e_{t}$

where $\left(1+\varphi_{1}-\psi_{1}\right)$ can be positive or negative, but regardless, it captures the relative effect of change of exchange rate to crude oil prices on petroleum product prices. Thus, one may write:

$\operatorname{petrol}_{t}^{D C}=\beta_{0}+\beta_{1}$ trend $+\beta_{2}$ crude $_{t}^{D C}+\beta_{3} e_{t}+u_{t}$

Here, the domestic currency value of domestic petroleum product prices (petrol ${ }^{D C}$ ) is determined by the domestic currency value of crude oil prices as well as exchange rate. The domestic currency value of crude oil prices (crude ${ }^{D C}$ ) is found by adding the logarithms of USDdenominated crude oil prices and exchange rate. Here, we also include the time trend to account for lower transportation costs due to higher efficiency of transportation vehicles and higher efficiency of distribution channels over time. $\beta_{3}$ captures the relative effect of exchange rate changes to crude oil price changes on petroleum product prices. ${ }^{4} \mathrm{~A}$

\footnotetext{
${ }^{4}$ Alternatively, we could include USD-denominated crude prices and exchange rates in the regression analysis. However, we would need to test whether the estimated parameters of these two variables are equal to each other. This test statistic is not readily available because it is also a function of the covariances of these two parameters (see Greene, 2008, ss. 53-56).
}

Table 1

The Engle-Granger cointegration test for pre-tax distribution prices.

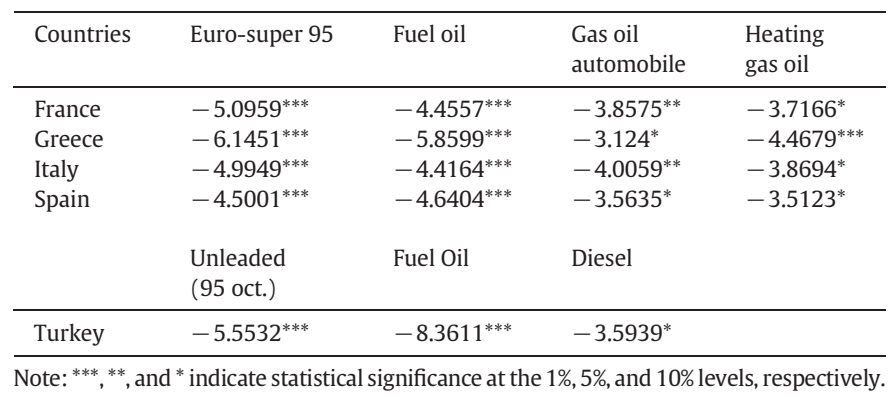

positive $\beta_{3}$ suggests that an increase in exchange rate increases petroleum products' pump prices more than the cost of crude oil prices in local currency does; a negative $\beta_{3}$ suggests that an increase in exchange rate increases petroleum products' pump prices less than the cost of crude oil prices in local currency does. ${ }^{5}$

If all the series have unit roots, these series should be cointegrated for a long-run relationship. Overall, unit root tests fail to reject the null of a unit root for world crude oil prices, exchange rates, and gasoline prices for each country. ${ }^{6}$ These statistics are not provided here to save space, but are available from the authors upon request.

Table 1 reports the Dickey-Fuller test statistics that correspond to the Engle-Granger cointegration tests. We consider various threevariable systems. We include various petroleum product prices, crude oil prices in local currency, and exchange rate, along with the constant term and time trend in each system. The test statistics suggest that the series are cointegrated at least at the $10 \%$ level of significance. Thus, we estimate Eq. (6), and Table 2 reports these estimated coefficients for this equation. The estimated coefficients of crude oil prices in local currency (crude $e^{D C}$ ) are always positive and statistically significant. These estimated coefficients are less than one and between 0.32 and 0.61. Campa and Goldberg (2005) report that even if there is a one percent increase in imported product prices in domestic currency, it almost always increases prices of products at the dock one percent (full pass-through of exchange rate to prices). This effect is smaller in consumer prices (incomplete pass-through) at the later stages of distribution channels. Thus, our results support their proposition.

On the estimated coefficient of the exchange rate, the estimated coefficients for all countries and products we consider are negative and statistically significant, except for unleaded (95 octane) for Turkey: it is positive, yet not statistically significant. This suggests that a one percent increase in crude oil prices increases petroleum product prices more than a one percent increase in exchange rate does. Even if crude oil is the most important input to the petroleum product production process, there are various other inputs, such as other types of chemicals, labor, and electricity. As the technology improves in the usage of these inputs, the output prices will be less affected (see Morrison, 1997). Since technological improvement on crude usage is limited compared to the non-crude inputs, crude prices will affect petroleum product prices more (see Concawe, 2007). Moreover, Burstein et al. (2003); Burstein et al. (2005); and Campa and Goldberg (2005) argue that the

\footnotetext{
5 Except for France, it is quite likely that crude oil prices and exchange rates are both exogenous to the system. Moreover, it is quite unlikely that pre-tax petroleum product prices affect world crude oil prices and exchange rate. Thus, we employ univariate but not multivariate analyses. We did estimate a VAR model but the confidence bands for the impulse responses had too wide a margin to make inferences. Crude oil prices and exchange rates were both exogenous to the system, thus estimating the model within a VAR framework might lead to overparamatization.

${ }^{6}$ We employed three unit root tests: ADF; Phillips and Perron (PP); and Kwiatkowski, Phillips, Schmidt, and Shin (KPSS). For the first two tests, the null hypothesis is the unit root, but for the last test the alternative is the unit root. We performed the test for the $\log$ levels and log first differences. Overall, we concluded that all series are integrated in order one, I(1).
} 
Table 2

Long-run relationship between Brent and domestic petroleum product pre-tax prices.

\begin{tabular}{|c|c|c|c|c|c|}
\hline Countries & Variables & Constant & Trend & Crude $^{\mathrm{DC}}$ & Exchange \\
\hline \multirow[t]{5}{*}{ France } & Euro-super 95 & $\begin{array}{l}5.6487^{* * *} \\
{[0.0000]}\end{array}$ & $\begin{array}{c}-0.1530^{* * * *} \\
{[0.0000]}\end{array}$ & $\begin{array}{c}0.4593^{* * * *} \\
{[0.0000]}\end{array}$ & $\begin{array}{c}-0.7794^{* * *} \\
{[0.0000]}\end{array}$ \\
\hline & Fuel oil & $\begin{array}{l}3.2097^{* * * *} \\
{[0.0000]}\end{array}$ & $\begin{array}{l}0.0272^{* * *} \\
{[0.0000]}\end{array}$ & $\begin{array}{c}0.4883^{* * *} \\
{[0.0000]}\end{array}$ & $\begin{array}{c}-0.8945^{* * *} \\
{[0.0000]}\end{array}$ \\
\hline & Gas oil & $6.5798^{* * *}$ & $-0.2378^{* * *}$ & $0.4602^{* * *}$ & $-0.9354^{* * *}$ \\
\hline & automobile & {$[0.0000]$} & {$[0.0000]$} & {$[0.0000]$} & {$[0.0000]$} \\
\hline & Heating gas oil & $\begin{array}{l}6.1108^{* * * *} \\
{[0.0000]}\end{array}$ & $\begin{array}{c}-0.1805^{* * *} \\
{[0.0000]}\end{array}$ & $\begin{array}{c}0.4322^{* * *} \\
{[0.0000]}\end{array}$ & $\begin{array}{c}-0.8827^{* * *} \\
{[0.0000]}\end{array}$ \\
\hline \multirow[t]{5}{*}{ Greece } & Euro-super 95 & $\begin{array}{l}6.1774^{* * * *} \\
{[0.0000]}\end{array}$ & $\begin{array}{c}-0.1849^{* * *} \\
{[0.0000]}\end{array}$ & $\begin{array}{c}0.4483^{* * * *} \\
{[0.0000]}\end{array}$ & $\begin{array}{c}-0.6607^{* * *} \\
{[0.0000]}\end{array}$ \\
\hline & Fuel oil & $\begin{array}{l}3.5673^{* * * *} \\
{[0.0000]}\end{array}$ & $\begin{array}{l}0.0205^{* * * *} \\
{[0.0000]}\end{array}$ & $\begin{array}{c}0.4439^{* * * *} \\
{[0.0000]}\end{array}$ & $\begin{array}{c}-0.8522^{* * *} \\
{[0.0000]}\end{array}$ \\
\hline & Gas oil & $6.6278^{* * * *}$ & $-0.1783^{* * *}$ & $0.3234^{* * * *}$ & $-0.9367^{* * * *}$ \\
\hline & automobile & {$[0.0000]$} & {$[0.0000]$} & {$[0.0000]$} & {$[0.0000]$} \\
\hline & Heating gas oil & $\begin{array}{l}6.7672^{* * * *} \\
{[0.0000]}\end{array}$ & $\begin{array}{c}-0.2273^{* * *} \\
{[0.0000]}\end{array}$ & $\begin{array}{c}0.4124^{* * * *} \\
{[0.0000]}\end{array}$ & $\begin{array}{c}-0.6613^{* * *} \\
{[0.0000]}\end{array}$ \\
\hline \multirow[t]{5}{*}{ Italy } & Euro-super 95 & $\begin{array}{l}4.9213^{* * * *} \\
{[0.0000]}\end{array}$ & $\begin{array}{c}-0.0593^{* * * *} \\
{[0.0000]}\end{array}$ & $\begin{array}{c}0.4607^{* * * *} \\
{[0.0000]}\end{array}$ & $\begin{array}{c}-0.3879^{* * * *} \\
{[0.0000]}\end{array}$ \\
\hline & Fuel oil & $\begin{array}{l}2.8703^{* * * *} \\
{[0.0000]}\end{array}$ & $\begin{array}{l}0.0609^{* * * *} \\
{[0.0000]}\end{array}$ & $\begin{array}{c}0.5458^{* * * *} \\
{[0.0000]}\end{array}$ & $\begin{array}{c}-0.4093^{* * *} \\
{[0.0000]}\end{array}$ \\
\hline & Gas oil & $5.6890^{* * *}$ & $-0.1293^{* * *}$ & $0.4679^{* * *}$ & $-0.4896^{* * *}$ \\
\hline & automobile & {$[0.0000]$} & {$[0.0000]$} & {$[0.0000]$} & {$[0.0000]$} \\
\hline & Heating gas oil & $\begin{array}{l}5.1551^{* * * *} \\
{[0.0000]}\end{array}$ & $\begin{array}{c}-0.0838^{* * *} \\
{[0.0000]}\end{array}$ & $\begin{array}{c}0.4685^{* * * *} \\
{[0.0000]}\end{array}$ & $\begin{array}{c}-0.5104^{* * *} \\
{[0.0000]}\end{array}$ \\
\hline \multirow[t]{5}{*}{ Spain } & Euro-super 95 & $\begin{array}{l}5.8708^{* * *} \\
{[0.0000]}\end{array}$ & $\begin{array}{c}-0.1493^{* * *} \\
{[0.0000]}\end{array}$ & $\begin{array}{c}0.4203^{* * * *} \\
{[0.0000]}\end{array}$ & $\begin{array}{c}-0.6967^{* * * *} \\
{[0.0000]}\end{array}$ \\
\hline & Fuel oil & $\begin{array}{l}4.2954^{* * * *} \\
{[0.0000]}\end{array}$ & $\begin{array}{c}-0.0434^{* * *} \\
{[0.0000]}\end{array}$ & $\begin{array}{c}0.4561^{* * *} \\
{[0.0000]}\end{array}$ & $\begin{array}{c}-0.5664^{* * *} \\
{[0.0000]}\end{array}$ \\
\hline & Gas oil & $6.5229^{* * * *}$ & $-0.2003^{* * *}$ & $0.3986^{* * *}$ & $-0.8907^{* * *}$ \\
\hline & automobile & {$[0.0000]$} & {$[0.0000]$} & {$[0.0000]$} & {$[0.0000]$} \\
\hline & Heating gas oil & $\begin{array}{l}5.9827^{* * * *} \\
{[0.0000]}\end{array}$ & $\begin{array}{c}-0.1929^{* * *} \\
{[0.0000]}\end{array}$ & $\begin{array}{c}0.4656^{* * * *} \\
{[0.0000]}\end{array}$ & $\begin{array}{c}-1.0393^{* * *} \\
{[0.0000]}\end{array}$ \\
\hline \multirow[t]{3}{*}{ Turkey } & $\begin{array}{l}\text { Unleaded (95 } \\
\text { oct.) }\end{array}$ & $\begin{array}{c}-4.4386^{* * *} \\
{[0.0000]}\end{array}$ & $\begin{array}{l}0.1912^{* * *} \\
{[0.0036]}\end{array}$ & $\begin{array}{c}0.5717^{* * * *} \\
{[0.0000]}\end{array}$ & $\begin{array}{r}0.0283 \\
{[0.6560]}\end{array}$ \\
\hline & Fuel oil & $\begin{array}{c}-21.2318^{* * *} \\
{[0.0000]}\end{array}$ & $\begin{array}{l}1.8216^{* * * *} \\
{[0.0000]}\end{array}$ & $\begin{array}{c}0.6091^{\text {**** }} \\
{[0.0000]}\end{array}$ & $\begin{array}{c}-0.3551^{* * * *} \\
{[0.0000]}\end{array}$ \\
\hline & Diesel & $\begin{array}{c}-5.7634^{* * *} \\
{[0.0000]}\end{array}$ & $\begin{array}{l}0.3566^{* * * *} \\
{[0.0004]}\end{array}$ & $\begin{array}{c}0.5306^{* * *} \\
{[0.0000]}\end{array}$ & $\begin{array}{c}-0.2444^{* *} \\
{[0.0117]}\end{array}$ \\
\hline
\end{tabular}

Note: $p$-values are reported in brackets under the corresponding coefficient. ${ }^{* * *},{ }^{* *}$, and ${ }^{*}$ indicate statistical significance at the $1 \%, 5 \%$, and $10 \%$ levels, respectively.

role of non-tradable cost is also important. Thus, the role of exchange rate (which is more than it is tied to crude prices) on petroleum product prices should be lower.

If these series have unit roots and are jointly cointegrated, then the specification may be written in an error correction form. Thus, one may write Eq. (6) as:

$$
\begin{aligned}
\Delta \text { petrol }_{t}^{D C}= & \alpha_{0}+\alpha^{E C M} u_{t-1} \\
& +\sum_{i=0}^{m} \alpha_{i}^{c} \Delta \text { crude } e_{t-i}^{D C}+\sum_{i=0}^{m} \alpha_{i}^{e} \Delta e_{t-i}+\sum_{i=1}^{m} \alpha_{i}^{d} \Delta \text { petrol }_{t-i}^{D C}+\omega_{t}
\end{aligned}
$$

where $\Delta$ is for the first difference operator. Here, $u_{t-1}$ is the lag value of the residual term of $6 . u_{t-1}$ captures the deviation of petroleum product prices from their long-run equilibrium from the previous period and $\alpha^{E C M}$ is the estimated parameter for the error correction term. $m$ is the lag length, $\alpha_{i}^{c}$ captures the short-run adjustment terms to crude oil prices in local currency, $\alpha_{i}^{e}$ is for the short-run adjustment terms to exchange rate, $\alpha_{i}^{d}$ is for the lagged dependent variable. Table 3 reports the estimated coefficients for Eq. (7).

The estimated coefficient for $u_{t-1}$ and $\alpha^{E C M}$ is expected to be negative because higher (or lower) domestic product prices from a longrun equilibrium would lead to lower (or higher) oil product prices to correct the error made from the previous period. The estimated coefficients for $u_{t-1}$ are all negative and statistically significant, which implies that an increase in domestic petroleum product prices above the longrun equilibrium suggested by Eq. (6), $u_{t}$, will decrease domestic petroleum product prices in the next period. Similarly, a decrease in domestic petroleum product prices below the long-run equilibrium implies that domestic petroleum product prices will increase in the next period. The estimated coefficients of the Euro-super 95 or unleaded (95 octane) specifications are the highest in absolute value for all countries but Spain. The estimated coefficients for fuel oil are the lowest for France, Greece, and Spain. Therefore, the adjustment for those countries can be considered the lowest.

If one looks at the estimated coefficients for $\Delta c r u d e_{t-i}^{\mathrm{DC}}$ for all countries and petroleum products, they tend to be positive and statistically significant. This finding suggests that an increase in crude prices increases petroleum product prices in local currency in the short run. If one looks at the estimated coefficients for $\Delta e_{t-i}$, there are a number of positive as well as negative coefficients. These negative coefficients tend to concentrate on the fourth lag. We will elaborate on this issue in the next paragraph, but this result suggests that as exchange rate increases and its cost effect is accounted for through crude oil price increases in local currency, it increases petroleum product prices earlier and the effect of exchange rate decreases over time. The estimated coefficients for $\Delta$ petrol $_{t-i}$ tend to be negative, which suggests that a change in petroleum product prices tends to die out over time. However, these coefficients do not reveal whether exchange rate or crude prices are affected differently than petroleum product prices.

To assess the relative effect of exchange rate versus crude oil prices on petroleum products in the short run we estimate the coefficient for the exchange rate: $\alpha_{i}^{e}$. The positive coefficient for $\alpha_{i}^{e}$ suggests that a one percent increase in exchange rate increases petroleum products more than a one percent increase in crude oil prices does for the $i^{\text {th }}$ period. Table 4 reports the cumulative sum of the estimates of the change in exchange rate coefficients $\sum \alpha_{i}^{e}$ to capture what the total short-run effect of exchange rate changes relative to crude oil price changes on petroleum products at the $n^{\text {th }}$ period would be. We estimate the model with four lags; however, the effects of exchange rate and crude oil prices may persist for more than four periods (one month). Thus, we estimate the model with eight lags when we analyze the short-run effects of these two variables on petroluem product prices. ${ }^{7}$ Specifically, for Period 0 we report the $\alpha_{0}^{e}$ estimate, for Period 1 we report the $\alpha_{0}^{e}+\alpha_{1}^{e}$ estimate, for Period 2 we report the $\alpha_{0}^{e}+\alpha_{1}^{e}+\alpha_{2}^{e}$ estimate, etc., to calculate the accumulated effect of exchange rate changes on petroleum products relative to crude oil price increases.

Table 4 reveals that all the statistically significant coefficients at the $10 \%$ level are positive. This result suggests that in the short run, a one percent increase in exchange rate increases petroleum product prices more than a one percent increase in crude oil prices does. This is parallel to findings of Asplund et al. (2000) for Sweden, ${ }^{8}$ and the result makes sense: first, refineries purchase their crude oil with forward contracts and might be immune to spot crude oil price fluctuations. However, unless agents particularly hedge against foreign exchange rate volatility with forward/future contracts, the exchange rate sensitivity of petroleum products should be higher. Thus, agents do respond to exchange rate changes more than they respond to crude oil price changes in the short run. Second, within the Rotemberg (1982) framework, where prices are set in an uncompetitive environment with a flexible markup and changing prices are costly to a firm (due to changing menu prices and lost reputation), firms will be reluctant to adjust their retail prices as input prices change. For inputs with a higher price volatility, firms will be more hesitant to increase their output prices when input prices increase than they will be to increase their inputs with a lower price volatility. Inputs with a higher price volatility (in our case, crude oil) may mean a lower input price in the future; thus, firms will be

\footnotetext{
${ }^{7}$ We also estimate the model with four lags and the estimates are mostly parallel with the eight-lag model.

${ }^{8}$ The specification employed by Asplund et al. (2000) is similar to our short-run analysis (they use the first-differenced series within a single equation framework); however, they did not search for the asymmetric effects of exchange rate changes and crude oil price changes on petroleum products explicitly and did not provide the test statistics. Although they did not elaborate on the asymmetry issue, their estimates reported in Table 4 reveal that cost increases from exchange rates increase more than those from crude.
} 
Table 3

Error correction specification for pre-tax prices.

\begin{tabular}{|c|c|c|c|c|c|c|c|c|c|c|c|c|c|c|c|c|c|}
\hline Countries & Variables & $\alpha_{0}$ & $\alpha_{0}^{c}$ & $\alpha_{1}^{c}$ & $\alpha_{2}^{c}$ & $\alpha_{3}^{c}$ & $\alpha_{4}^{c}$ & $\alpha_{0}^{e}$ & $\alpha_{1}^{e}$ & $\alpha_{2}^{e}$ & $\alpha_{3}^{e}$ & $\alpha_{4}^{e}$ & $\alpha_{1}^{d}$ & $\alpha_{2}^{d}$ & $\alpha_{3}^{d}$ & $\alpha_{4}^{d}$ & $u_{t-1}$ \\
\hline \multirow[t]{6}{*}{ France } & Euro-super 95 & $\begin{array}{l}0.0001 \\
{[0.6916]}\end{array}$ & $\begin{array}{c}0.0803^{* * *} \\
{[0.0000]}\end{array}$ & $\begin{array}{l}0.0492^{* * *} \\
{[0.0001]}\end{array}$ & $\begin{array}{c}0.0936^{* * *} \\
{[0.0000]}\end{array}$ & $\begin{array}{c}0.0994^{* * *} \\
{[0.0000]}\end{array}$ & $\begin{array}{c}0.0547^{* * *} \\
{[0.0000]}\end{array}$ & $\begin{array}{r}0.0470 \\
{[0.2713]}\end{array}$ & $\begin{array}{r}0.0518 \\
{[0.2268]}\end{array}$ & $\begin{array}{c}-0.0459 \\
{[0.2844]}\end{array}$ & $\begin{array}{c}0.0646 \\
{[0.1321]}\end{array}$ & $\begin{array}{c}-0.0207 \\
{[0.6269]}\end{array}$ & $\begin{array}{c}-0.0697^{* * *} \\
{[0.0022]}\end{array}$ & $\begin{array}{c}-0.0528^{* *} \\
{[0.0186]}\end{array}$ & $\begin{array}{c}-0.0216 \\
{[0.3298]}\end{array}$ & $\begin{array}{c}-0.0126 \\
{[0.5685]}\end{array}$ & $\begin{array}{c}-0.0135^{* * *} \\
{[0.0000]}\end{array}$ \\
\hline & Fuel oil & 0.0002 & $0.1170^{* * *}$ & $0.1161^{* * *}$ & $0.1364^{* * *}$ & $0.0778^{* * *}$ & 0.0115 & $0.1872^{* * *}$ & 0.0228 & -0.0435 & 0.0061 & -0.0327 & $-0.0802^{* * *}$ & $-0.0760^{* * *}$ & -0.0286 & -0.0124 & $-0.0146^{* * *}$ \\
\hline & & {$[0.4820]$} & {$[0.0000]$} & {$[0.0000]$} & {$[0.0000]$} & {$[0.0000]$} & {$[0.4846]$} & {$[0.0005]$} & {$[0.6754]$} & [0.4239] & [0.9112] & {$[0.5446]$} & [0.0005] & {$[0.0011]$} & {$[0.2084]$} & {$[0.5802]$} & {$[0.0000]$} \\
\hline & Gas oil automobile & $\begin{array}{l}0.0000 \\
{[0.9529]}\end{array}$ & $\begin{array}{c}0.0747^{* * * *} \\
{[0.0000]}\end{array}$ & $\begin{array}{l}0.0446^{* * * *} \\
{[0.0000]}\end{array}$ & $\begin{array}{c}0.0801^{* * * *} \\
{[0.0000]}\end{array}$ & $\begin{array}{c}0.0753^{* * * *} \\
{[0.0000]}\end{array}$ & $\begin{array}{c}0.0446^{* * * *} \\
{[0.0000]}\end{array}$ & $\begin{array}{r}0.0229 \\
{[0.4932]}\end{array}$ & $\begin{array}{r}0.0184 \\
{[0.5820]}\end{array}$ & $\begin{array}{c}-0.0362 \\
{[0.2804]}\end{array}$ & $\begin{array}{c}0.0561^{*} \\
{[0.0942]}\end{array}$ & $\begin{array}{c}-0.0807^{* *} \\
{[0.0156]}\end{array}$ & $\begin{array}{c}-0.0888^{* * *} \\
{[0.0001]}\end{array}$ & $\begin{array}{c}-0.0770^{* * *} \\
{[0.0006]}\end{array}$ & $\begin{array}{l}-0.0307 \\
{[0.1654]}\end{array}$ & $\begin{array}{c}-0.0148 \\
{[0.5033]}\end{array}$ & $\begin{array}{c}-0.0054^{* * *} \\
{[0.0070]}\end{array}$ \\
\hline & Heating gas oil & 0.0001 & $0.0875^{* * * *}$ & $0.0586^{* * *}$ & $0.0732^{* * * *}$ & $0.0616^{* * * *}$ & $0.0249^{* *}$ & $0.0594^{* *}$ & 0.0270 & -0.0187 & 0.0139 & -0.0432 & $-0.0880^{* * * *}$ & $-0.0846^{* * * *}$ & $-0.0686^{* * * *}$ & -0.0117 & $-0.0077^{* * *}$ \\
\hline & & {$[0.7545]$} & {$[0.0000]$} & {$[0.0000]$} & {$[0.0000]$} & {$[0.0000]$} & {$[0.0145]$} & {$[0.0751]$} & {$[0.4209]$} & {$[0.5769]$} & {$[0.6787]$} & {$[0.1946]$} & {$[0.0001]$} & {$[0.0002]$} & {$[0.0023]$} & [0.6032] & {$[0.0002]$} \\
\hline \multirow[t]{7}{*}{ Greece } & Euro-super 95 & 0.0001 & $0.0357^{* * * *}$ & -0.0046 & $0.0475^{* * *}$ & $0.0661^{* * *}$ & $0.0463^{* * *}$ & 0.0329 & $0.0722^{* *}$ & -0.0332 & -0.0221 & -0.0550 & -0.0302 & -0.0167 & 0.0069 & -0.0028 & $-0.0122^{* * *}$ \\
\hline & & {$[0.6672]$} & {$[0.0016]$} & {$[0.6870]$} & {$[0.0000]$} & {$[0.0000]$} & [0.0001] & {$[0.3938]$} & {$[0.0623]$} & {$[0.3926]$} & [0.5679] & {$[0.1540]$} & {$[0.1837]$} & {$[0.4573]$} & {$[0.7581]$} & {$[0.9016]$} & {$[0.0$} \\
\hline & Fuel oil & 0.0002 & $0.0845^{* * * *}$ & $0.0938^{* * *}$ & $0.1066^{* * *}$ & $0.1017^{* * *}$ & $0.0468^{* * *}$ & $0.1843^{* * * *}$ & -0.0157 & 0.0101 & $0.0907^{*}$ & $-0.0848^{*}$ & -0.0 & $-0.0524^{* *}$ & -0.0213 & -0.0128 & -0.0 \\
\hline & & $\begin{array}{l}{[0.4650]} \\
0.0001\end{array}$ & $\begin{array}{l}{[0.0000]} \\
0.0266^{* * *}\end{array}$ & $\begin{array}{l}{[0.0000]} \\
-0.0221^{* *}\end{array}$ & $\begin{array}{l}{[0.0000]} \\
0.0298^{* * *}\end{array}$ & $\begin{array}{l}{[0.0000]} \\
0.0359^{* * *}\end{array}$ & [0.0028] & $\begin{array}{r}{[0.0004]} \\
0.0280\end{array}$ & $\begin{array}{c}{[0.7633]} \\
0.0701^{* * *}\end{array}$ & $\begin{array}{l}0.8457] \\
-0.0381\end{array}$ & $\begin{array}{l}{[0.0815]} \\
-0.0108\end{array}$ & $\begin{array}{l}{[0.1008]} \\
-0.0469 *\end{array}$ & $\begin{array}{l}{[0.0023]} \\
-0.0304\end{array}$ & $\begin{array}{l}{[0.0204]} \\
-0.0232\end{array}$ & $\begin{array}{c}{[0.3415]} \\
0.0050\end{array}$ & $\begin{array}{l}{[0.5639]} \\
-0.0157\end{array}$ & {$[0.0$} \\
\hline & Gas oil automobile & $\begin{array}{l}0.0001 \\
{[0.5578]}\end{array}$ & $\begin{array}{l}0.0266^{6} \\
{[0.0004]}\end{array}$ & $\begin{array}{l}-0.0221^{*} \\
{[0.0038]}\end{array}$ & $\begin{array}{l}0.0298 \\
{[0.0001]}\end{array}$ & $\begin{array}{l}0.0359^{*} \\
{[0.0000]}\end{array}$ & $\begin{array}{l}0.0255^{\prime} \\
{[0.0009]}\end{array}$ & $\begin{array}{r}0.0280 \\
{[0.2764]}\end{array}$ & $\begin{array}{l}0.0701^{* 14} \\
{[0.0067]}\end{array}$ & $\begin{array}{l}-0.0381 \\
{[0.1411]}\end{array}$ & $\begin{array}{l}-0.0108 \\
{[0.6755]}\end{array}$ & $\begin{array}{l}-0.0469^{*} \\
{[0.0686]}\end{array}$ & $\begin{array}{l}-0.0304 \\
{[0.1811]}\end{array}$ & $\begin{array}{l}-0.0232 \\
{[0.3038]}\end{array}$ & $\begin{array}{l}0.0050 \\
{[0.8240]}\end{array}$ & $\begin{array}{l}-0.0157 \\
{[0.4875]}\end{array}$ & $\begin{array}{c}-0.0064 \\
{[0.0000]}\end{array}$ \\
\hline & Heating gas oil & 0.0001 & $0.0232^{*}$ & $-0.0303^{* *}$ & $0.0407^{* * *}$ & $0.0225^{*}$ & $0.0390^{* * *}$ & 0.0692 & $0.1721^{* * * *}$ & $-0.0864^{* *}$ & 0.0498 & -0.0448 & -0.0055 & -0.0081 & 0.0185 & 0.0014 & $-0.012^{* * *}$ \\
\hline & & {$[0.6352]$} & {$[0.0862]$} & {$[0.0268]$} & {$[0.0030]$} & {$[0.0997]$} & {$[0.0044]$} & [0.1349] & {$[0.0002]$} & {$[0.0645]$} & {$[0.2865]$} & [0.3347] & [0.8098] & {$[0.7205]$} & 109] & {$[0.9518]$} & {$[0.0000]$} \\
\hline \multirow[t]{8}{*}{ Italy } & Euro-super 95 & 0.0002 & $0.0698^{* * * *}$ & $0.0447^{* * *}$ & $0.0651^{* * *}$ & $0.0599^{* * *}$ & $0.0394^{* * *}$ & -0.0243 & 0.0183 & $-0.0607^{*}$ & 0.0267 & -0.0538 & $-0.0599^{* * * *}$ & $-0.0571^{* *}$ & $-0.0393^{*}$ & -0.0186 & $-0.0143^{* * *}$ \\
\hline & & {$[0.4689]$} & & & {$[0.0000]$} & {$[0.0000]$} & {$[0.0002]$} & {$[0.4859]$} & [0.5998] & & {$[0.4446]$} & & {$[0.0091]$} & {$[0.0$} & & & {$[0$.} \\
\hline & Fuel oil & 0.0002 & $0.1005^{* * *}$ & $0.0996^{* * *}$ & $0.1068^{* * *}$ & $0.0632^{* * *}$ & $0.0396^{* * *}$ & $0.1502^{* * *}$ & 0.0184 & 323 & 0.0595 & 0480 & $-0.0770^{* * *}$ & $-0.0736^{* * *}$ & 308 & 0186 & $-0.0129^{* * *}$ \\
\hline & & {$[0.4072]$} & & & & {$[0.0000]$} & [0.0047] & {$[0.0$} & [0.6907] & & {$[0.1$} & & & & & & \\
\hline & Gas oil automobile & 0.0001 & $0.0732^{* * *}$ & $0.0276^{* * *}$ & $0.0533^{* * *}$ & $0.0459^{* * *}$ & $0.0293^{* * *}$ & -0.0144 & 0.0135 & -0.0239 & 0.0153 & $-0.0480^{*}$ & $-0.0636^{* * *}$ & $-0.0612^{* * *}$ & 231 & -0.0181 & $-0.0093^{* * * *}$ \\
\hline & & {$[0.7404]$} & {$[0.0000]$} & {$[0.0021]$} & {$[0.0000]$} & {$[0.0000]$} & & {$[0.6276]$} & {$[0.6493]$} & {$[0.4211]$} & {$[0.6064]$} & & {$[0.0053]$} & {$[0.0069]$} & {$[0.3$} & & {$[0.0002]$} \\
\hline & Heating gas oil & & $0.0770^{* * * *}$ & $0.0601^{* * *}$ & $0.0782^{* * *}$ & $0.0394^{* * *}$ & $0.0203^{* *}$ & 0.0141 & 0.0032 & $487^{*}$ & 0.0083 & $-0.0577^{* *}$ & $-0.0959^{* * *}$ & $-0.1049^{* * *}$ & $-0.0622^{* * *}$ & $-0.0441^{* *}$ & $-0.0109^{* * *}$ \\
\hline & & & {$[0.0000]$} & {$[0.0$} & {$[0.0000]$} & {$[0.0000]$} & & {$[0.6296]$} & [0.9124] & & {$[0.77$} & & & {$[0.0$} & {$[0.0$} & & 0] \\
\hline \multirow[t]{8}{*}{ Spain } & Euro-super 95 & 0.0001 & $0.0645^{* * * *}$ & $0.0308^{* * *}$ & $0.0756^{* * * *}$ & $0.0570^{* * * *}$ & $0.0353^{* * *}$ & 0.0503 & 0.0307 & $-0.0671^{* *}$ & 0.0446 & -0.0261 & $-0.0584^{* *}$ & $-0.0547^{* *}$ & -0.0180 & -0.0151 & $-0.0102^{* * * *}$ \\
\hline & & {$[0.6$} & {$[0.0000]$} & {$[0.00$} & {$[0.0$} & {$[0.0000]$} & & & [0.3465] & & & & & {$[0.0$} & & {$[0.5$} & \\
\hline & Fuel oil & 0.0044 & 0.0395 & 0.0435 & $0.1017^{*}$ & 0.0832 & $0.1038^{* *}$ & -0.0950 & -0.0098 & -0.2641 & -0.0211 & -0.1706 & $-0.3275^{* * * *}$ & $-0.2761^{* * *}$ & $0.1364^{* * *}$ & 0.0177 & $-0.0228^{* * * *}$ \\
\hline & & {$[0.0476]$} & & 10 & & & & {$[05680]$} & {$[0.9$} & & $1088-3-1$ & & & & & & \\
\hline & Gas oil automobile & 0.0000 & $0.0613^{* * *}$ & $0.0291^{* * *}$ & $0.0630^{* * * *}$ & $0.0409^{* * *}$ & $0.0220^{* * * *}$ & 0.0429 & 0.0218 & -0.0360 & 0.0273 & -0.0194 & $-0.0687^{* * *}$ & $-0.0745^{* * *}$ & -0.0208 & -0.0162 & $-0.0057^{* * *}$ \\
\hline & & {$[0.9100]$} & [n 00001 & {$[0.0003]$} & {$[0.0000]$} & {$[0.0000]$} & [0.0063] & [0.1046] & {$[0.4115]$} & {$[0.1757]$} & {$[0.3058]$} & {$[0.4637]$} & {$[0.0026]$} & 1000111 & [0 3557$]$ & [0 10721] & (00010 \\
\hline & Heating gas oil & 0.0001 & $0.0611^{* * *}$ & -0.0095 & $0.0549^{* * * *}$ & $0.0358^{* * * *}$ & $0.0288^{* * *}$ & 0.0195 & $0.0708^{* *}$ & -0.0461 & 0.0354 & $-0.0771^{* *}$ & -0.0337 & $-0.0418^{* *}$ & 0.0058 & -0.0058 & $-0.0082^{* * *}$ \\
\hline & & {$[0.5845]$} & (5) & {$[0.3318]$} & {$[0.0000]$} & (0) & [0.0035] & 05510 & {$[0.0316]$} & {$[01628]$} & 10?02011 & 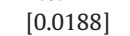 & [0.1376] & {$[0.0$} & [0.7981] & 200 & (1) \\
\hline \multirow[t]{5}{*}{ Turkey } & Unleaded (95 oct) & 0.0008 & $0.1680^{* * *}$ & $0.1372^{* * *}$ & $0.1348^{* * *}$ & 0.0333 & $0.1228^{* * *}$ & 0.0832 & 0.1100 & 0.0018 & 0.0140 & $-0.1923^{* *}$ & 0.0497 & $-0.1140^{* *}$ & 0.0066 & -0.0740 & $-0.0827^{* * *}$ \\
\hline & & & & & & {$[0.3446]$} & & {$[0.2946]$} & {$[0.1584]$} & {$[0.9819]$} & {$[0.8540]$} & & & & & {$[0.1503]$} & \\
\hline & Fuel oil & 0.0040 & 0.0535 & $0.1733^{* * *}$ & $0.2355^{* * * *}$ & $0.1825^{* * * *}$ & $0.1831^{* * * *}$ & 0.1123 & -0.0431 & -0.0558 & -0.0311 & -0.1778 & $-0.4973^{* * *}$ & $-0.3923^{* * *}$ & 0.0798 & -0.0109 & $-0.0655^{* *}$ \\
\hline & & & & & & & & [0.3300] & & & & {$[0.1121]$} & & & 1. 00501 & & \\
\hline & Diesel & $\begin{array}{l}0.0012 \\
{[0.4453]}\end{array}$ & $\begin{array}{c}0.0636^{* *} \\
{[0.0486]}\end{array}$ & $\begin{array}{l}0.0954^{* * *} \\
{[0.0049]}\end{array}$ & $\begin{array}{c}0.1375^{* * *} \\
{[0.0000]}\end{array}$ & $\begin{array}{l}0.0840^{* *} \\
{[0.0140]}\end{array}$ & $\begin{array}{l}-0.0129 \\
{[0.7052]}\end{array}$ & $\begin{array}{l}0.2138^{* * *} \\
{[0.0080]}\end{array}$ & $\begin{array}{l}-0.0998 \\
{[0.2120]}\end{array}$ & $\begin{array}{c}-0.0460 \\
{[0.5675]}\end{array}$ & $\begin{array}{c}-0.1353^{*} \\
{[0.0874]}\end{array}$ & $\begin{array}{r}0.0845 \\
{[0.2841]}\end{array}$ & $\begin{array}{l}-0.1148^{* *} \\
{[0.0515]}\end{array}$ & $\begin{array}{l}-0.1074^{* *} \\
{[0.0664]}\end{array}$ & $\begin{array}{c}0.0501 \\
{[0.3702]}\end{array}$ & $\begin{array}{c}-0.0451 \\
{[0.4056]}\end{array}$ & $\begin{array}{c}-0.0457^{* * *} \\
{[0.0057]}\end{array}$ \\
\hline
\end{tabular}

Note: $p$-values are reported in brackets under the corresponding coefficient. ${ }^{* * *},{ }^{* *}$, and ${ }^{*}$ indicate statistical significance at the $1 \%, 5 \%$, and $10 \%$ levels, respectively. 
Table 4

Cumulative short-run effect of excess of exchange rate to crude oil price changes on pre-tax petroleum product prices.

\begin{tabular}{|c|c|c|c|c|c|c|c|c|c|c|}
\hline Countries & Variables & 0 & 1 & 2 & 3 & 4 & 5 & 6 & 7 & 8 \\
\hline \multirow[t]{8}{*}{ France } & \multirow[t]{2}{*}{ Euro-super 95} & $0.0737^{*}$ & $0.0931^{*}$ & 0.0616 & $0.1387^{*}$ & 0.1340 & 0.1157 & 0.0357 & 0.0170 & 0.0165 \\
\hline & & [0.0699] & {$[0.0947]$} & [0.3691] & {$[0.0815]$} & {$[0.1302]$} & {$[0.2354]$} & {$[0.7345]$} & {$[0.8791]$} & [0.8886] \\
\hline & \multirow[t]{2}{*}{ Fuel oil } & $0.1771^{* * *}$ & $0.1930^{* * *}$ & 0.1381 & 0.1479 & 0.1042 & 0.2069 & 0.1923 & 0.1568 & 0.0964 \\
\hline & & {$[0.0010]$} & {$[0.0092]$} & [0.1307] & [0.1633] & {$[0.3775]$} & {$[0.1113]$} & [0.1708] & {$[0.2950]$} & [0.5426] \\
\hline & \multirow[t]{2}{*}{ Gas oil automobile } & 0.0370 & 0.0280 & -0.0004 & 0.0488 & -0.0104 & -0.0478 & -0.0989 & -0.1086 & -0.1221 \\
\hline & & [0.2443] & {$[0.5210]$} & [0.9933] & {$[0.4357]$} & [0.8809] & {$[0.5336]$} & {$[0.2336]$} & [0.2207] & [0.1934] \\
\hline & \multirow[t]{2}{*}{ Heating gas oil } & $0.0757^{* *}$ & $0.0820^{*}$ & 0.0740 & 0.0903 & 0.0573 & 0.0052 & -0.0316 & -0.0663 & -0.1049 \\
\hline & & [0.0197] & {$[0.0661]$} & {$[0.1785]$} & [0.1577] & {$[0.4207]$} & {$[0.9467]$} & {$[0.7085]$} & {$[0.4635]$} & [0.2730] \\
\hline \multirow[t]{8}{*}{ Greece } & \multirow[t]{2}{*}{ Euro-super 95} & 0.0397 & $0.0867^{*}$ & 0.0625 & 0.0597 & 0.0194 & 0.0452 & -0.0222 & -0.0507 & 0.0150 \\
\hline & & {$[0.2721]$} & {$[0.0804]$} & [0.3057] & [0.3990] & [0.8057] & [0.6017] & [0.8126] & [0.6113] & [0.8869] \\
\hline & \multirow[t]{2}{*}{ Fuel oil } & $0.1757^{* * *}$ & $0.1599^{* *}$ & $0.1660^{*}$ & $0.2595^{* *}$ & 0.1716 & 0.1850 & 0.1276 & 0.1036 & 0.0604 \\
\hline & & [0.0006] & [0.0232] & {$[0.0558]$} & {$[0.0101]$} & {$[0.1274]$} & {$[0.1352]$} & {$[0.3401]$} & {$[0.4680]$} & [0.6884] \\
\hline & \multirow{2}{*}{ Gas oil automobile } & 0.0279 & $0.0756^{* *}$ & 0.0341 & 0.0290 & 0.0013 & 0.0242 & -0.0285 & -0.0394 & -0.0092 \\
\hline & & [0.2428] & {$[0.0214]$} & [0.3995] & [0.5361] & [0.9787] & {$[0.6746]$} & [0.6464] & [0.5529] & [0.8950] \\
\hline & \multirow[t]{2}{*}{ Heating gas oil } & 0.0545 & $0.2228^{* * *}$ & $0.1370^{*}$ & $0.1836^{* *}$ & 0.1392 & 0.1335 & 0.0637 & 0.1198 & 0.0976 \\
\hline & & {$[0.2371]$} & {$[0.0004]$} & [0.0792] & [0.0427] & [0.1679] & {$[0.2293]$} & [0.5949] & [0.3490] & [0.4703] \\
\hline \multirow[t]{8}{*}{ Italy } & \multirow[t]{2}{*}{ Euro-super 95} & 0.0104 & 0.0005 & -0.0411 & -0.0000 & -0.0360 & 0.0486 & 0.0500 & 0.0330 & -0.0094 \\
\hline & & {$[0.7510]$} & [0.9909] & [0.4584] & [0.9999] & [0.6148] & {$[0.5372]$} & [0.5572] & {$[0.7160]$} & [0.9213] \\
\hline & \multirow[t]{2}{*}{ Fuel oil } & $0.1454^{* * *}$ & $0.1615^{* *}$ & 0.1197 & $0.1821^{* *}$ & 0.1316 & $0.1841^{*}$ & 0.1918 & 0.1283 & 0.0943 \\
\hline & & [0.0016] & {$[0.0111]$} & [0.1268] & [0.0453] & [0.1937] & [0.0984] & [0.1111] & [0.3173] & [0.4866] \\
\hline & \multirow[t]{2}{*}{ Gas oil automobile } & 0.0071 & -0.0165 & -0.0323 & -0.0176 & -0.0519 & 0.0024 & 0.0022 & -0.0080 & -0.0172 \\
\hline & & [0.7998] & {$[0.6717]$} & {$[0.5003]$} & {$[0.7505]$} & [0.4019] & {$[0.9711]$} & {$[0.9758]$} & [0.9185] & [0.8361] \\
\hline & \multirow[t]{2}{*}{ Heating gas oil } & 0.0348 & 0.0222 & -0.0201 & -0.0195 & -0.0583 & -0.0273 & -0.0337 & -0.0313 & -0.0535 \\
\hline & & [0.2225] & {$[0.5710]$} & {$[0.6771]$} & {$[0.7274]$} & {$[0.3494]$} & {$[0.6905]$} & [0.6492] & {$[0.6921]$} & [0.5221] \\
\hline \multirow[t]{8}{*}{ Spain } & \multirow[t]{2}{*}{ Euro-super 95} & $0.0710^{* *}$ & $0.0917^{* *}$ & 0.0353 & 0.0879 & 0.0803 & 0.0271 & -0.0201 & -0.0494 & -0.0947 \\
\hline & & [0.0153] & {$[0.0228]$} & {$[0.4766]$} & [0.1272] & {$[0.2100]$} & {$[0.7004]$} & [0.7915] & [0.5417] & [0.2683] \\
\hline & \multirow[t]{2}{*}{ Fuel oil } & -0.0266 & $0.1636^{* *}$ & $0.1705^{* *}$ & $0.2056^{* *}$ & $0.2168^{* *}$ & $0.3013^{* *}$ & $0.2688^{* *}$ & 0.2282 & $0.2507^{*}$ \\
\hline & & {$[0.5950]$} & {$[0.0177]$} & {$[0.0451]$} & {$[0.0374]$} & {$[0.0489]$} & {$[0.0130]$} & [0.0406] & [0.1035] & [0.0908] \\
\hline & \multirow[t]{2}{*}{ Gas oil automobile } & $0.0573^{* *}$ & $0.0630^{*}$ & 0.0326 & 0.0575 & 0.0604 & 0.0111 & -0.0174 & -0.0551 & -0.0831 \\
\hline & & {$[0.0202]$} & {$[0.0632]$} & {$[0.4348]$} & [0.2363] & {$[0.2642]$} & {$[0.8519]$} & [0.7867] & [0.4227] & [0.2532] \\
\hline & \multirow[t]{2}{*}{ Heating gas oil } & 0.0245 & 0.0643 & 0.0205 & 0.0413 & -0.0163 & -0.0390 & -0.1226 & -0.1329 & -0.1451 \\
\hline & & [0.4199] & [0.1249] & [0.6910] & {$[0.4910]$} & [0.8069] & {$[0.5956]$} & [0.1233] & {$[0.1178]$} & [0.1064] \\
\hline \multirow[t]{6}{*}{ Turkey } & \multirow[t]{2}{*}{ Unleaded (95 oct.) } & 0.0877 & 0.1601 & 0.2045 & 0.1943 & 0.0693 & -0.0085 & 0.1108 & 0.0229 & -0.1020 \\
\hline & & [0.2642] & [0.1316] & [0.1363] & [0.2268] & {$[0.7002]$} & {$[0.9644]$} & [0.5894] & [0.9157] & [0.6552] \\
\hline & \multirow[t]{2}{*}{ Fuel oil } & 0.0462 & 0.1568 & 0.1114 & 0.1736 & 0.1110 & 0.0332 & 0.1203 & 0.1573 & 0.0581 \\
\hline & & {$[0.6786]$} & [0.2999] & {$[0.5703]$} & [0.4527] & {$[0.6708]$} & {$[0.9070]$} & [0.6969] & {$[0.6344]$} & [0.8700] \\
\hline & \multirow[t]{2}{*}{ Diesel } & $0.2111^{* * *}$ & 0.1618 & 0.0762 & -0.0257 & 0.0861 & -0.0222 & 0.1678 & 0.1728 & 0.1486 \\
\hline & & [0.0091] & [0.1433] & [0.5971] & [0.8794] & {$[0.6512]$} & [0.9136] & [0.4445] & [0.4562] & [0.5454] \\
\hline
\end{tabular}

Note: $p$-values are reported in brackets under the corresponding coefficient. ${ }^{* * *},{ }^{* *}$, and ${ }^{*}$ indicate statistical significance at the $1 \%, 5 \%$, and $10 \%$ levels, respectively.

more hesitant to adjust their output prices (petroleum product prices here) to crude prices than to implement cost increases due to exchange rate increases. The descriptive statistics suggest that the volatility of crude price changes is higher than both exchange rates we consider (the standard deviation of crude percent change was 2.3288 , the standard error of USD to Euro exchange rate change was 0.6766, and the USD to Turkish Lira exchange rate was 2.0869).

Third, firms will be more reluctant to change their petroleum product prices if they think input price changes are temporary rather than permanent. To test this hypothesis, we perform the Lo and MacKinlay $(1988,1989)$ variance ratio test. The test statistics are reported in Table 5 for two to eight periods for crude oil prices in USD as well as for Euro-USD and TL-USD exchange rates. Except for the fourth period for Turkish exchange rates, the variance ratio test reveals a higher variance ratio for both exchange rates than for crude oil prices. None of the test statistics at $10 \%$ rejects the null of martingale, but martingale is rejected at $10 \%$ in the fourth, fifth, sixth, seventh, and eighth periods for crude. Thus, test statistics support the proposition that shocks to exchange rates are more persistent and agents adjust pump prices faster if input prices increase due to exchange rate than if they increase due to crude price increases. Thus, firms may respond more slowly to a change in crude price changes than to a change in exchange rate in the short run.

When we observe selected countries, Turkey has the lowest shortrun asymmetry. Close values of standard errors in exchange rate changes and crude oil price changes (compared to other countries) also support this proposition. This finding makes sense because dollarization is high and the exchange rate is volatile in Turkey; thus, the markets have evolved such that the price adjustment mechanism is fastest there. When we compare the number of petroleum products in which exchange rate asymmetry has been observed, Greece has the highest degree of short-run exchange rate asymmetry.

There is a set of studies that suggests retail gasoline prices respond asymmetrically to cost increases compared to cost decreases. Brown and Yucel (2000) interpret the existence of asymmetry to the monopolistic structure of oil and petroleum products. Johnson (2002) promotes the search cost to explain asymmetric prices. Borenstein and Shepard (1996) and Borenstein et al. (1997) emphasize different sources of asymmetries at different stages of the distribution chain. To incorporate

Table 5

Lo and MacKinlay variance ratio tests results.

\begin{tabular}{|c|c|c|c|}
\hline \multirow[t]{2}{*}{ Periods } & \multicolumn{3}{|c|}{ Variance ratio test statistics } \\
\hline & Crude oil prices & Euro-USD exchange rate & TL-USD exchange rate \\
\hline 2 & $\begin{array}{l}0.9364 \\
{[0.1641]}\end{array}$ & 1.0050 [0.8657] & $\begin{array}{l}1.0207 \\
{[0.8052]}\end{array}$ \\
\hline 3 & $\begin{array}{l}0.8969 \\
{[0.1039]}\end{array}$ & $\begin{array}{l}0.9848 \\
{[0.7227]}\end{array}$ & $\begin{array}{l}0.9168 \\
{[0.532]}\end{array}$ \\
\hline 4 & $\begin{array}{l}0.8653^{*} \\
\quad[0.0742]\end{array}$ & $0.9694 \quad[0.5616]$ & $\begin{array}{l}0.8487 \\
{[0.3843]}\end{array}$ \\
\hline 5 & $\begin{array}{l}0.8245^{* *} \\
{[0.0433]}\end{array}$ & 0.9662 & $\begin{array}{l}0.8548 \\
{[0.4807]}\end{array}$ \\
\hline 6 & $\begin{array}{l}0.8036^{* *} \\
{[0.0464]}\end{array}$ & $\begin{array}{l}0.9603 \\
{[0.5597]}\end{array}$ & $\begin{array}{l}0.8749 \\
{[0.5893]}\end{array}$ \\
\hline 7 & $\begin{array}{l}0.7934^{*} \\
\quad[0.0599]\end{array}$ & $\begin{array}{l}0.9631 \\
\end{array}$ & $\begin{array}{l}0.8850 \\
{[0.6502]}\end{array}$ \\
\hline 8 & $\begin{array}{l}0.7931^{*} \\
\quad[0.0846]\end{array}$ & $\begin{array}{l}0.9728 \\
{[0.7408]}\end{array}$ & $\begin{array}{l}0.9032 \\
{[0.7225]}\end{array}$ \\
\hline
\end{tabular}

Note: $p$-values are reported in brackets under the corresponding coefficient. ${ }^{* * *},{ }^{* *}$, and ${ }^{*}$ indicate statistical significance at the $1 \%, 5 \%$, and $10 \%$ levels, respectively. 
Table 6

Error correction specification for pre-tax prices.

\begin{tabular}{|c|c|c|c|c|c|c|c|c|c|c|c|c|c|c|c|c|c|c|c|}
\hline Countries & Variables & $u_{t-1}^{+}$ & $u_{t-1}^{-}$ & $\alpha_{0}$ & $\alpha_{0}^{c}$ & $\alpha_{1}^{c}$ & $\alpha_{2}^{c}$ & $\alpha_{3}^{c}$ & $\alpha_{4}^{c}$ & $\alpha_{0}^{e}$ & $\alpha_{1}^{e}$ & $\alpha_{2}^{e}$ & $\alpha_{3}^{e}$ & $\alpha_{4}^{e}$ & $\alpha_{1}^{d}$ & $\alpha_{2}^{d}$ & $\alpha_{3}^{d}$ & $\alpha_{4}^{d}$ & $H_{0}$ \\
\hline \multirow[t]{7}{*}{ France } & Euro 95 & $\begin{array}{c}-0.0111 \\
{[0.0507]}\end{array}$ & $\begin{array}{c}-0.0161 \\
{[0.0057]}\end{array}$ & $\begin{array}{r}0.0003 \\
{[0.5288]}\end{array}$ & $\begin{array}{l}0.0803^{* * *} \\
{[0.0000]}\end{array}$ & $\begin{array}{l}0.0487^{* * *} \\
{[0.0001]}\end{array}$ & $\begin{array}{c}0.0932^{* * *} \\
{[0.0000]}\end{array}$ & $\begin{array}{l}0.0990^{* * *} \\
{[0.0000]}\end{array}$ & $\begin{array}{c}0.0544^{* * *} \\
{[0.0000]}\end{array}$ & $\begin{array}{r}0.0469 \\
{[0.2722]}\end{array}$ & $\begin{array}{r}0.0521 \\
{[0.2242]}\end{array}$ & $\begin{array}{c}-0.0456 \\
{[0.2881]}\end{array}$ & $\begin{array}{r}0.0649 \\
{[0.1306]}\end{array}$ & $\begin{array}{l}-0.0202 \\
{[0.6349]}\end{array}$ & $\begin{array}{c}-0.0689 \\
{[0.0026]}\end{array}$ & $\begin{array}{l}-0.0521 \\
{[0.0203]}\end{array}$ & $\begin{array}{c}-0.0212 \\
{[0.3382]}\end{array}$ & $\begin{array}{c}-0.0124 \\
{[0.5743]}\end{array}$ & $\begin{array}{c}0.2656 \\
{[0.6063}\end{array}$ \\
\hline & Fuel oil & -0.0194 & -0.0102 & 0.0008 & $0.1167^{* * * *}$ & $0.1156^{* * * *}$ & $0.1362^{* * *}$ & $0.0778^{* * *}$ & 0.0113 & $0.1890^{* * *}$ & 0.0247 & -0.0412 & 0.0080 & -0.0309 & -0.0799 & -0.0759 & $\begin{array}{l}{[.0 .302]} \\
-0.0289\end{array}$ & -0.0131 & 0.5615 \\
\hline & & {$[0.0074]$} & {$[0.1305]$} & {$[0.2242]$} & $.0000]$ & {$[0.0000]$} & {$[0.0000]$} & {$[0.0000]$} & [0.4945] & {$[0.0005]$} & {$[0.6500]$} & {$[0.4494]$} & {$[0.8831]$} & {$[0.5678]$} & {$[0.0006]$} & {$[0.0011]$} & {$[0.2046]$} & [0.5595] & {$[0.4537]$} \\
\hline & Gas auto & -0.0027 & -0.0104 & 0.0004 & $0.0736^{* * *}$ & $0.0437^{* * *}$ & $0.0793^{* * *}$ & & $0.0441^{* * *}$ & 0.0243 & 0.0199 & -0.0341 & $0.0578^{*}$ & $-0.0782^{* *}$ & -0.0897 & -0.0783 & -0.0318 & -0.0162 & 1.1921 \\
\hline & & {$[0.3798]$} & [0.0367] & {$[0.2485]$} & & & & & {$[0.0000]$} & [0.4661] & {$[0.5517]$} & & {$[0.0848]$} & [0.0191] & [0.0001] & [0.0005] & [0.1509] & [0.4634] & {$[0.2750]$} \\
\hline & Heating & & -0.0136 & 0.0004 & $0.0868^{* * *}$ & $0.0580^{* * *}$ & $0.0728^{* * *}$ & $0.0613^{* * * *}$ & $0.0246^{* *}$ & $0.0604^{*}$ & 0.0284 & & 0.0154 & -0.0413 & -0.0886 & -0.0854 & -0.0693 & -0.0127 & 1.6986 \\
\hline & Furn 09 & {$[0.2046]$} & {$[0.0064]$} & {$[0.2847]$} & {$[0.0000]$} & {$[0.0000]$} & {$[0.0000]$} & {$[0.0000]$} & {$[0.0159]$} & [0.0701] & {$[0.3967]$} & [0.6120] & [0.6465] & {$[0.2155]$} & {$[0.0001]$} & [0.0002] & {$[0.0021]$} & [0.5723] & [0.1926] \\
\hline \multirow{7}{*}{ Greece } & Euro 95 & $\begin{array}{l}-0 . \\
{[0.00}\end{array}$ & $\begin{array}{r}-0 \\
{[0.08}\end{array}$ & $\begin{array}{l}0.000 \\
{[0.05}\end{array}$ & $\begin{array}{l}0.034 \\
{[0.00}\end{array}$ & $\begin{array}{l}-0.6 \\
{[0.58}\end{array}$ & $\begin{array}{c}0.0460^{* * *} \\
{[0.0001]}\end{array}$ & $\begin{array}{l}0.0646^{* * *} \\
{[0.0000]}\end{array}$ & $\begin{array}{c}0.0448^{* * *} \\
{[0.0001]}\end{array}$ & $\begin{array}{r}0.0348 \\
{[0.3664]}\end{array}$ & $\begin{array}{c}0.0749^{*} \\
{[0.0531]}\end{array}$ & $\begin{array}{l}-0 . c \\
{[0.43}\end{array}$ & $\begin{array}{l}-0.0196 \\
{[0.6123]}\end{array}$ & {$\left[\begin{array}{c}-0 . \\
{[0.18}\end{array}\right.$} & $\begin{array}{r}-0 \\
{[0.21}\end{array}$ & $\begin{array}{r}-0 \\
{[0.49}\end{array}$ & $\begin{array}{c}0.0079 \\
{[0.7240]}\end{array}$ & $\begin{array}{l}-[0.0025] \\
{[0.9098]}\end{array}$ & $\begin{array}{c}0.2738 \\
{[0.6008]}\end{array}$ \\
\hline & Fuel oil & -0.0211 & -0.0116 & 0.0005 & $0.0841^{* * * *}$ & $0.0931^{* * *}$ & $0.1059^{* * *}$ & $0.1012^{* * *}$ & $0.0464^{* * *}$ & $0.1855^{* * * *}$ & -0.0142 & 0.0117 & $0.0921^{*}$ & -0.0833 & -0.0696 & -0.0523 & -0.0214 & -0.0132 & 0.6325 \\
\hline & & {$[0.0044]$} & [0.0672] & {$[0.3723]$} & {$[0.0000]$} & {$[0.0000]$} & & {$[0.0000]$} & [0.0031] & [0.0003] & [0.7854] & [0.8227] & {$[0.0770]$} & [0.1077] & [0.0024] & {$[0.0207]$} & [0.3394] & & {$[0.4$} \\
\hline & Gas auto & -0.0063 & -0.0066 & $0.0004^{*}$ & $0.0257^{* * *}$ & $-0.0229^{* * *}$ & $0.0289^{* * *}$ & $0.0352^{* * *}$ & $0.0247^{* * *}$ & 0.0295 & $0.0718^{* * * *}$ & -0.0359 & -0.0089 & $-0.0444^{*}$ & -0.0315 & -0.0248 & 0.0033 & -0.0175 & 0.0029 \\
\hline & & {$[0.0107]$} & {$[0.0877]$} & {$[0.0885]$} & {$[0.0007]$} & {$[0.0027]$} & {$[0.0002]$} & {$[0.0000]$} & {$[0.0013]$} & [0.2503] & {$[0.0055]$} & & {$[0.7308]$} & & & & & & \\
\hline & Heating & & & 0.00 & 0.0211 & $-0.0338^{* *}$ & 0.03 & 0.0193 & 0.03 & 0.0696 & $0.1754^{* * * *}$ & -0 & 0.0534 & 389 & -0 & 078 & 0.0186 & 016 & 0.0278 \\
\hline & & {$[0.0$} & {$[0.0$} & {$[0.0308]$} & [0.1178] & {$[0.0$} & 72] & {$[0.1588]$} & {$[0.0$} & [0.1322] & {$[0.0002]$} & & {$[0.2525]$} & {$[0$.} & {$[0.8$} & {$[0.7$} & & & {$[0.8$} \\
\hline \multirow[t]{7}{*}{ Italy } & Euro 95 & $-0.0120^{*}$ & $-0.0169^{* *}$ & -0.0002 & $0.0701^{* * *}$ & $0.0443^{* * *}$ & $0.0647^{* * *}$ & $0.0596^{* * *}$ & $0.0393^{* * *}$ & -0.0249 & 0.0185 & -0 & 0.0266 & -0.0539 & 599 & $1^{* *}$ & $33^{*}$ & 184 & 70 \\
\hline & & {$[0.0661]$} & {$[0.01$} & {$[0.6874]$} & & {$[0.0000]$} & {$[0.0000]$} & 1000 & {$[0.0002]$} & {$[0.4$} & {$[0.5958]$} & & [0.4461] & & & & & & \\
\hline & Fuel oil & $-0.0143^{* *}$ & -0.0113 & 0.0006 & $0.1002^{* * *}$ & $0.0996^{* * * *}$ & $0.1068^{* * *}$ & $0.0633^{* * *}$ & $0.0394^{* * *}$ & $0.1513^{* * *}$ & 0.0188 & -0.0313 & 0.0602 & -0.0472 & -0.0770 & $-0.0739^{* * *}$ & -0.0314 & -0.0193 & 0.0599 \\
\hline & & {$[0.0267]$} & {$[0.12$} & {$[0.2500]$} & & {$[0.0000]$} & & & & {$[0.0$} & [0.6839] & & [0.1942] & {$[0.3$} & {$[0.00$} & & & & \\
\hline & Gas auto & -0.0 & & & & 276 & $0.0534^{* * *}$ & 0.0 & $3^{* * *}$ & & 6 & & 54 & & & $5^{* * *}$ & 33 & 85 & 0.2 \\
\hline & & & & & & & (2) & & & & & & & & & & & & \\
\hline & Heating & $\begin{array}{c}-0.0116^{* * *} \\
{[0.0065]}\end{array}$ & $\begin{array}{l}-0.0099^{*} \\
{[0.0931]}\end{array}$ & $\begin{array}{r}0.0004 \\
{[0.2399]}\end{array}$ & $\begin{array}{c}0.0767^{* * *} \\
000001\end{array}$ & $\begin{array}{l}0.0599^{* * * *} \\
{[0.0000}\end{array}$ & $\begin{array}{c}0.0781^{* * * *} \\
{[0.0000]}\end{array}$ & $0.0394^{* * * *}$ & $0.0202^{* *}$ & $\begin{array}{r}0.0146 \\
{[0.6191]}\end{array}$ & $\begin{array}{r}0.0036 \\
{[0.9029]}\end{array}$ & $\begin{array}{l}-0.0483^{*} \\
{[0.006]}\end{array}$ & 0.0085 & $\begin{array}{l}-0.0572^{*} \\
{[0.0502]}\end{array}$ & -0.0969 & $-0.1059^{* * *}$ & $-0.0629^{* * *}$ & $-0.0449^{* *}$ & 0.0359 \\
\hline \multirow{8}{*}{ Spain } & Euro 95 & $-0.0105^{* *}$ & $-0.0099^{* *}$ & 0.0005 & $0.0642^{* * * *}$ & $\begin{array}{l}0.00000 \\
0.0299^{* * *}\end{array}$ & $\begin{array}{l}{[0.0000]} \\
0.0747^{* * *}\end{array}$ & $\begin{array}{l}{[0.0000]} \\
0.0563^{* * *}\end{array}$ & $\begin{array}{l}{[0.0248]} \\
0.0347^{* * *}\end{array}$ & $\begin{array}{r}{[0.6191]} \\
0.0505\end{array}$ & $\begin{array}{l}.9029] \\
0.0317\end{array}$ & $\begin{array}{c}{[0.1006]} \\
-0.0659^{* *}\end{array}$ & 0.0459 & $\begin{array}{c}{[0.0502]} \\
-0.0248\end{array}$ & $\begin{array}{c}{[0.0000]} \\
-0.0567\end{array}$ & $\begin{array}{l}{[0.0000]} \\
-0.0536^{* *}\end{array}$ & $\begin{array}{l}{[0.0063]} \\
-0.0176\end{array}$ & $\begin{array}{l}{[0.0478]} \\
-0.0152\end{array}$ & $\begin{array}{l}0.8497] \\
0.0055\end{array}$ \\
\hline & & & & {$[0.1671]$} & & 1 & & & & & & & & & & & & & \\
\hline & Fuel oil & $-0.0282^{* * *}$ & $-0.0175^{* * *}$ & -0.0044 & 0.0441 & 0.0712 & $0.1259^{* *}$ & $0.1008^{* * *}$ & $0.1154^{*}$ & -0.0963 & -0.0385 & $-0.3284^{* *}$ & -0.0862 & $-0.2637^{*}$ & -0.3057 & $-0.2870^{* * *}$ & $0.1059^{*}$ & 0.0098 & 0.8 \\
\hline & & {$[0.0$} & {$[0.0$} & {$[0.2097]$} & 57] & & & & & {$[0.55$} & {$[0.8126]$} & {$[0.0$} & [0.5957] & {$[0.1$} & {$[0.00$} & {$[0.0$} & {$[0.0$} & {$[0.8$} & {$[0.3475]$} \\
\hline & Gas auto & & & $0.0006^{* *}$ & & & 0.06 & & & $0.0451^{*}$ & & & 0.0304 & & & & & 01 & \\
\hline & & & & & & & & & & & & & & & & & & & \\
\hline & Heating & -0. & $-0.0101^{* *}$ & $0.0006^{* *}$ & $0.0598^{* * *}$ & -0.0108 & $0.0536^{* * *}$ & $0.0350^{* * *}$ & $0.0280^{* * *}$ & 0.0222 & $0.0741^{* *}$ & -0.0422 & 0.0384 & -0.0733 & -0.0364 & -0.0 & 0.0024 & -0.0094 & 0.2927 \\
\hline & & & & & & & & & & & 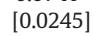 & & (2) & & & & & & \\
\hline \multirow[t]{6}{*}{ Turkey } & Unleaded & -0.0072 & $-0.1346^{* * *}$ & -0.0034 & $0.1632^{* * * *}$ & $0.1465^{* * *}$ & $0.1485^{* * *}$ & 0.0469 & $0.1298^{* * *}$ & 0.0771 & 0.1117 & 0.0122 & 0.0134 & -0.1801 & 0.0387 & $-0.1189^{* *}$ & -0.0033 & -0.0824 & 2.5892 \\
\hline & & & & & & & & & & {$[0.32$} & & & & & & & & & \\
\hline & Fuel oil & -0 & -0.1 & 0.0013 & 0.0581 & $0.1793^{* * *}$ & $0.2416^{* * *}$ & $0.1867^{* * *}$ & $0.1868^{* * *}$ & 0.0989 & -0.0480 & -0 & -0 & -0.1800 & $-0.4971^{* * *}$ & $-0.3959^{* * *}$ & 0.0726 & -0.0136 & 1.2 \\
\hline & & & & {$[0.7488]$} & & & & & & & {$[0$} & {$[0.574$} & & {$[0.1081]$} & {$[0.0000]$} & {$[0.0000]$} & {$[0.2097]$} & {$[0.7966]$} & {$[0.2731]$} \\
\hline & Diesel & -0.0053 & $-0.0981^{* * *}$ & -0.0028 & $0.0691^{* *}$ & $0.0990^{* * *}$ & $0.1403^{* * *}$ & $0.0899^{* * *}$ & -0.0072 & $0.2184^{* * * *}$ & -0.0962 & -0.0444 & $-0.1436^{*}$ & 0.0785 & -0.1018 & $-0.0964^{*}$ & 0.0537 & -0.0468 & $2.8872^{*}$ \\
\hline & & & & {$[0.3216]$} & .0327] & {$[0.0035]$} & {$[0.0000]$} & {$[0.0087]$} & {$[0.8341]$} & {$[0.0067]$} & {$[0.2274]$} & {$[0.5798]$} & {$[0.0695]$} & .3188] & {$[0.0856]$} & & {$[0.3356]$} & {$[0.3864]$} & {$[0.0903]$} \\
\hline
\end{tabular}

Note: $p$-values are reported in brackets under the corresponding coefficient. ${ }^{* * *},{ }^{* *}$, and ${ }^{*}$ indicate statistical significance at the $1 \%, 5 \%$ and $10 \%$ levels, respectively. 
asymmetric effects, following Granger and Lee (1989) we adopt a set of specifications to be estimated. We first consider an asymmetry by Eq. (8):

$$
\begin{aligned}
\operatorname{spetrol}_{t}^{D C}= & \alpha_{0}+\alpha^{E C M^{+}} u_{t-1}^{+}+\alpha^{E C M^{-}} u_{t-1}^{-} \\
& +\sum_{i=0}^{p} \alpha_{i}^{c} \Delta \text { crude }_{t-i}^{D C}+\sum_{i=0}^{p} \alpha_{i}^{e} \Delta e_{t-i}+\sum_{i=1}^{p} \alpha_{i}^{d} \Delta \text { petrol }_{t-i}^{D C}+\omega_{t}
\end{aligned}
$$

$u_{t}^{+}= \begin{cases}u_{t} & \text { if } \quad u_{t} \geq 0 \\ 0 & \text { otherwise }\end{cases}$

and

$u_{t}^{-}=\left\{\begin{array}{ll}u_{t} & \text { if } u_{t}<0 \\ 0 & \text { otherwise }\end{array}\right.$.

Table 6 reports the estimated coefficients for Eq. (8). The estimated coefficients for positive and negative residuals $\left(u_{t-1}^{-}\right.$and $\left.u_{t-1}^{+}\right)$are always negative and at least one of $u_{t-1}^{+}$or $u_{t-1}^{-}$is statistically significant. Thus, the error correction term seems to be working. We did not interpret but we do report the coefficients for $\Delta c r u d e_{t-i}^{\mathrm{DC}}, \Delta e_{t-i}$ and $\Delta$ petrol $_{t-i}^{\mathrm{DC}}$. The interpretation gathered for these coefficients in Table 3 is robust. The last column reports the test statistics for the null hypothesis, that the coefficients for $u_{t-1}^{+}$are the same as $u_{t-1}^{-}$. None of the test statistics is statistically significant at the $10 \%$ level except for the Turkish diesel case, and Turkish diesel is also significant at the nine percent level. Thus, we may claim that we could not find a statistically significant asymmetry for crude oil price and exchange rate increases versus decreases on petroleum product prices, which is parallel to the findings of Borenstein et al. (1997). For this reason, we did not pursue this avenue further by calculating the cumulative effects of exchange rate changes relative to crude prices on petroleum product prices.

\section{Summary}

The purpose of this article is to study the relative pass-through effects of these two costs on petroleum product prices. Evidence from a set of Northern Mediterranean countries suggests that a one percent increase in exchange rate (depreciation) increases petroleum product prices more than a one percent increase in crude oil prices does in the short run. However, in the long run, a one percent increase in exchange rate increases petroleum product prices less than a one percent increase in crude oil prices does.

\section{Appendix A. Supplementary data}

Supplementary data to this article can be found online at http://dx. doi.org/10.1016/j.econmod.2014.07.019.

\section{References}

Asplund, M., Eriksson, R., Friberg, R., 2000. Price adjustments by a gasoline retail chain. Scand. J. Econ. 102, 101-121.

Bachmeier, L., Griffin, J., 2003. New evidence on asymmetric gasoline price responses. Rev. Econ. Stat. 85, 772-776.

Bacon, R.W., 1991. Rockets and feathers: the asymmetric speed of adjustment of U.K. retail gasoline prices to cost changes. Energy Econ. 13, 211-218.

Balke, N.S., Brown, S.P.A., Yucel, M., 1998. Crude oil and gasoline prices: an asymmetric relationship? Econ. Financ. Policy Rev. Q1, 2-11.

Borenstein, S., Shepard, A., 1996. Dynamic pricing in retail gasoline markets. RAND J. Econ. 27, 429-451.

Borenstein, S., Cameron, A.C., Gilbert, R., 1997. Do gasoline prices respond asymmetrically to crude oil price changes. Q. J. Econ. 112, 305-339.

Brown, S.P.A., Yucel, M.K., 2000. Gasoline and crude oil prices: why the asymmetry? Econ. Financ. Policy Rev. Q3, 23-29.

Burstein, A.T., Neves, J.C., Rebelo, S., 2003. Distribution costs and real exchange-rate dynamics during exchange-rate-based`stabilizations. J. Monet. Econ. 50, 1189-1214.

Burstein, A.T., Eichenbaum, M., Rebelo, S., 2005. Large devaluations and the real exchange rate. J. Polit. Econ. 113, 742-784.

Campa, J.M., Goldberg, L.S., 2005. Exchange rate pass-through into import prices. Rev. Econ. Stat. 87, 679-690.

Chou, K., 2012. Price adjustment in Taiwan retail gasoline market. Int. J. Econ. Financ. 4, $132-140$.

Concawe, 2007. Refinery technology support group. Oil refining in the EU in 2015. Refinery Technology Support Group Report No. 1(07).

Galeotti, M., Lanza, A., Manera, M., 2003. Rockets and feathers revisited: an international comparison on European gasoline markets. Energy Econ. 25, 175-190.

Godby, R., Lintner, A.M., Stegnos, T., Wandschneider, B., 2000. Testing for asymmetric pricing in the Canadian retail gasoline market. Energy Econ. 22, 349-368.

Granger, C.W.J., Lee, T.H., 1989. Investigation of production sales and inventory relationships, using multicointegration and non-symmetric error correction models. J. Appl. Econ. 4, 145-159.

Greene, W., 2008. Econometric Analysis, Sixth edition. Prentice Hall, USA.

Johnson, R., 2002. Search costs, lags and prices at the pump. Rev. Ind. Organ. 20, 33-50.

Kirchgassner, G., Kubler, K., 1992. Symmetric or asymmetric price adjustments in the oil market: an empirical analysis of the relations between international and domestic prices in the Federal Republic of Germany. Energy Econ. 14, 171-185.

Lo, A.W., MacKinlay, A.C., 1988. Stock market prices do not follow random walks: evidence from a simple specification test. Rev. Financ. Stud. 1, 41-66.

Lo, A.W., MacKinlay, A.C., 1989. The size and power of the variance ratio test in finite samples. J. Econ. 40, 203-238.

Morrison, C.J., 1997. Economic performance, cost economies and pricing behaviour in the US and Australian meat products industries. Aust. J. Agric. Resour. Econ. 41, 361-383. Norman, Donald A., David, Shin, 1991. Price adjustment in gasoline and heating oil markets. American Petroleum Institute Research Study No. 60, Washington.

Pindyck, R., 2001. The dynamics of commodity spot and future markets: a primer. Energy J. $22,1-29$.

Radchenko, S., 2005. Oil price volatility and the asymmetric response of gasoline prices to oil price increases and decreases. Energy Econ. 27, 708-730.

Reilly, B., Witt, R., 1998. Petrol price asymmetries revisited. Energy Econ. 20, 297-308. Rotemberg, J.J., 1982. Sticky prices in the United States. J. Polit. Econ. 90, 1187-1211. 